\title{
Article
}

\section{Transcriptional Stress Induces Chromatin Relocation of the Nucleotide Excision Repair Factor XPG}

\author{
Claudia Scalera ${ }^{1,+}$, Giulio Ticli ${ }^{1,2} \mathbb{D}^{\mathbb{D}}$, Ilaria Dutto ${ }^{1}\left(\mathbb{D}\right.$, Ornella Cazzalini ${ }^{3}$, Lucia A. Stivala ${ }^{3, *}$ \\ and Ennio Prosperi ${ }^{1, *(D)}$
}

1 Istituto di Genetica Molecolare "Luigi Luca Cavalli-Sforza", Consiglio Nazionale Delle Ricerche (CNR), Via Abbiategrasso 207, 27100 Pavia, Italy; C.Scalera@imb-mainz.de (C.S.); giulio.ticli@igm.cnr.it (G.T.); ilaria.dutto@gmail.com (I.D.)

2 Dipartimento di Biologia e Biotecnologie, Università di Pavia, Via Ferrata 9, 27100 Pavia, Italy

3 Dipartimento di Medicina Molecolare, Università di Pavia, Via Ferrata 9, 27100 Pavia, Italy; ornella.cazzalini@unipv.it

* Correspondence: luciaanna.stivala@unipv.it (L.A.S.); ennio.prosperi@igm.cnr.it (E.P.); Tel.: +39-0382-986267 (E.P.)

† Present address: Institute of Molecular Biology (IMB), Ackermannweg 4, 55128 Mainz, Germany.

check for updates

Citation: Scalera, C.; Ticli, G.; Dutto, I.; Cazzalini, O.; Stivala, L.A.; Prosperi, E. Transcriptional Stress Induces Chromatin Relocation of the Nucleotide Excision Repair Factor XPG. Int. J. Mol. Sci. 2021, 22, 6589. https://doi.org/10.3390/ijms22126589

Academic Editor: Thomas Liehr

Received: 4 March 2021

Accepted: 18 June 2021

Published: 19 June 2021

Publisher's Note: MDPI stays neutral with regard to jurisdictional claims in published maps and institutional affiliations.

Copyright: (c) 2021 by the authors. Licensee MDPI, Basel, Switzerland. This article is an open access article distributed under the terms and conditions of the Creative Commons Attribution (CC BY) license (https:/ / creativecommons.org/licenses/by/ $4.0 /)$.

\begin{abstract}
Endonuclease XPG participates in nucleotide excision repair (NER), in basal transcription, and in the processing of RNA/DNA hybrids (R-loops): the malfunction of these processes may cause genome instability. Here, we investigate the chromatin association of XPG during basal transcription and after transcriptional stress. The inhibition of RNA polymerase II with 5,6-dichloro$1-\beta$-D-ribofuranosyl benzimidazole (DRB), or actinomycin D (AD), and of topoisomerase I with camptothecin (CPT) resulted in an increase in chromatin-bound XPG, with concomitant relocation by forming nuclear clusters. The cotranscriptional activators p300 and CREB-binding protein (CREBBP), endowed with lysine acetyl transferase (KAT) activity, interact with and acetylate XPG. Depletion of both KATs by RNA interference, or chemical inhibition with C646, significantly reduced XPG acetylation. However, the loss of KAT activity also resulted in increased chromatin association and the relocation of XPG, indicating that these processes were induced by transcriptional stress and not by reduced acetylation. Transcription inhibitors, including C646, triggered the R-loop formation and phosphorylation of histone H2AX ( $\gamma-\mathrm{H} 2 \mathrm{AX})$. Proximity ligation assay (PLA) showed that XPG colocalized with R-loops, indicating the recruitment of the protein to these structures. These results suggest that transcriptional stress-induced XPG relocation may represent recruitment to sites of R-loop processing.
\end{abstract}

Keywords: XPG endonuclease; chromatin association; p300/CREBBP; R loop; acetylation; genome instability

\section{Introduction}

Endonuclease XPG, belongs to the flap endonuclease (FEN) family of enzymes that cleave DNA at structure-specific sites [1,2]. For example, UV-induced DNA lesions significantly distort the double helix, generating a structure that, following DNA opening by DNA helicases, is cleaved by XPG at the $3^{\prime}$ end, and by XPF at the $5^{\prime}$ end during the nucleotide excision repair (NER) process [3-5]. DNA incision activity by XPG is missing in a group of patients with xeroderma pigmentosum (XP) with mutations in the ERCC5 gene coding for XPG protein [6,7]. This inactivation leads to a cancer-prone phenotype, which is also accompanied by severe deficiency in development, and by neurological disorder when XPG mutations are associated with Cockayne syndrome (CS), to produce a complex disease (XPG/CS) [8-10].

In addition to its fundamental role in NER, XPG participates in basal transcription, being recruited to the promoters of RNA II polymerase (pol II)-dependent genes [11,12]. 
This process facilitates chromatin looping and DNA demethylation reactions requiring the catalytic activity of XPG for the accurate transcription of these genes $[13,14]$, thus explaining developmental defects induced by XPG mutations [11,12]. Furthermore, it was also shown to interact with WRN helicase and to play a role in homologous recombination (HR) induced by replication stress [15,16].

During transcription, RNA-DNA hybrids (R-loops) can be physiologically generated, but they may also result from defective transcription elongation of RNA, thus providing a source of genome instability [17]. XPG together with XPF endonuclease, are involved in the processing of R-loops, which under certain conditions may result in the formation of double-strand breaks (DSBs) $[18,19]$. When R-loops are formed following DNA damage, their processing requires the activity of XPG with Rad52 in order to activate transcriptionassociated HR [20].

The binding to DNA, the association with other factors, and chromatin recruitment are important determinants for XPG activity [21-24]. In particular, the chromatin association of NER factors needs to be regulated in a timely manner during DNA repair, since excessive retention results in genotoxic stress $[25,26]$. In previous studies, we showed that the XPG protein was acetylated by the lysine acetyl transferases (KAT) p300 and CREBBP (CBP) after UV irradiation, and suggested that this post-translational modification could influence the chromatin association of XPG during NER [27]. In addition, XPG was shown to be degraded after damage through the interaction with the CRL4 ${ }^{\mathrm{Cd} t 2}$ ubiquitin ligase complex [28]. However, the mechanisms regulating XPG recruitment to chromatin and turnover during the process of basal transcription have not been specifically addressed yet.

In this study, we investigate the chromatin association of XPG during basal transcription and after transcription inhibition. We show that transcriptional stress induced by inhibitors of RNA polymerase (pol) I and II or topoisomerase I promote the chromatin accumulation of XPG in concomitance with its redistribution to nuclear clustered zones. In addition, we show that the loss of p300 and CBP KAT activity mediated by RNA interference or by chemical inhibitor C646 [29] resulted in XPG accumulation and relocalization, similarly to transcription inhibitors. Chromatin accumulation and clustered redistribution occurred in concomitance with R-loop formation and histone $\gamma$-H2AX appearance. Analysis with PLA showed that XPG was in close proximity to R-loops, suggesting that relocation induced by transcriptional stress represents the recruitment of XPG to R-loops sites.

\section{Results}

\subsection{Chromatin Association of XPG}

To analyze the XPG fraction that was chromatin-bound in the absence of DNA damage, we applied an in situ lysis procedure to detect chromatin-associated proteins by immunofluorescence [30]. In proliferating fibroblasts, XPG was previously shown to be present in S-phase cells [15]. Therefore, we analyzed the amount of chromatin-bound XPG under basal-growth conditions, and whether the distribution of this XPG fraction may be dependent on the cell-cycle phase. Immunofluorescence analysis was performed in proliferating HaCaT keratinocytes and in LF-1 fibroblasts. Fluorescence signals showed that high levels of the XPG protein were sometimes observed in concomitance with the presence of chromatin-bound PCNA (Figure 1A), which marks DNA replication foci under unperturbed conditions [30]. XPG association with chromatin may consistently occur in the $S$ phase [15]. Therefore, to more quantitatively assess the cell-cycle distribution of chromatin-associated XPG, immunofluorescence was analyzed by flow cytometry. The biparametric determination of immunofluorescence vs DNA content indicated that the chromatin-bound form of XPG increased from the G1 to the S phase, reaching the highest values in the G2/M compartment (Figure 1B), resembling the distribution of a housekeeping protein. A detergent-extraction procedure, followed by DNase I digestion, was performed both on HaCaT keratinocytes and primary human LF-1 fibroblasts to obtain the unbound-soluble and chromatin-bound forms of XPG for Western blot analysis [27]. 
Densitometry quantification of the bands indicated that the unbound fraction, referred to as detergent-soluble protein (Sol), was predominant, while the chromatin-bound $(\mathrm{Cb})$ form accounted for about $20-25 \%$ of the total XPG protein (Figure 1C). The fraction of the soluble vs the chromatin-bound form of PCNA, which is present only during the $\mathrm{S}$ phase in proliferating cells [30], is shown for comparison (Figure 1C).

A
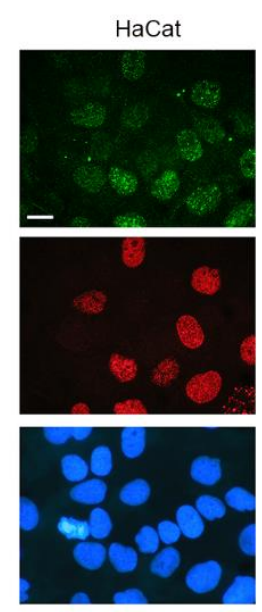

LF-1

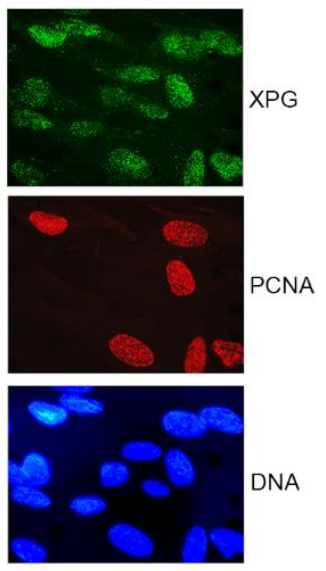

B
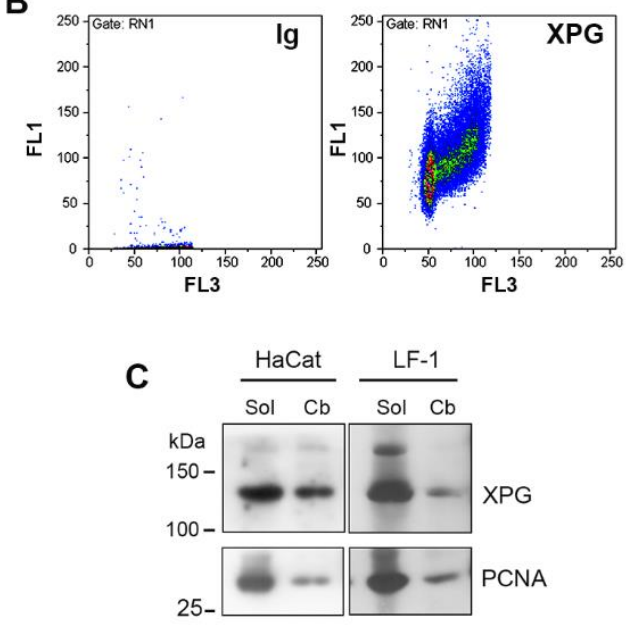

Figure 1. (A) Immunofluorescence of chromatin-bound form of XPG and PCNA proteins in HaCaT keratinocytes and in LF-1 fibroblasts. Cell grown on coverslips were subjected to in situ detergent extraction and fixed as described in Materials and Methods. XPG (green fluorescence) and PCNA (red fluorescence) shown with DNA (blue fluorescence). Scale bar $=10 \mu \mathrm{m}$. (B) Flow-cytometry dual-parameter analysis of cell-cycle distribution of chromatin-bound XPG in HaCaT cells. Immunofluorescence (FL1) was detected on cells stained with secondary antibody only (Ig), or with anti-XPG specific antibody, and counterstained with propidium iodide (PI) for DNA content (FL3). (C) Western blot analysis of detergent-soluble (Sol) and chromatin-bound ( $\mathrm{Cb}$ ) fractions of XPG and PCNA in HaCaT keratinocytes and LF-1 fibroblasts.

\subsection{Acetylation of Chromatin-Bound XPG}

As mentioned above, in our previous studies, XPG protein was acetylated after UV irradiation, but some modification appeared to be present even in the absence of DNA damage in a whole cell extract of LF-1 fibroblasts [27]. Therefore, to obtain information on the chromatin-bound fraction, we analyzed the acetylation of the XPG protein, and its interaction with p300/CBP in nuclear extracts of proliferating HaCaT cells. Immunoprecipitation assays were performed with an antibody to CBP, or with antiacetyl lysine (acK) antibody, of which the specificity was previously tested [31]. Results confirmed that the XPG protein was present in both samples, indicating that, in the fraction associated with chromatin, XPG interacted with CBP, and an acetylated form could be identified (Supplementary Figure S1).

To investigate whether the general inhibition of transcription would affect XPG acetylation, LF-1 cells were treated for $6 \mathrm{~h}$ with DRB, an inhibitor of RNA pol II, and after protein extraction, an immunoprecipitation (Ip) with the anti-acK antibody was performed. The specificity of the acK antibody was tested on untreated LF-1 cells by comparing the Ip of acetylated XPG in a cell extract in the presence of the HDAC inhibitors Na butyrate and Trichostatin A (TSA) vs an extract in which these were omitted. Another control was performed by incubating a parallel aliquot of cell extract with purified IgG in place of the acK antibody (Figure 2A). In samples incubated with DRB, Western blot analysis of XPG showed that, as compared with untreated controls, the inhibitor of RNA pol II did not abolish XPG acetylation since the modified form was immunoprecipitated from nuclear extracts of both samples. In addition, analysis of the input protein showed an increase in 
the amount of chromatin-bound XPG in the DRB-treated sample (Figure 2B). To further validate this result, HEK cells expressing HA-tagged XPG protein [12] were similarly treated with DRB or exposed to UV radiation, and nuclear extracts were immunoprecipitated with anti-acK antibody, as above. Results showed that HA-tagged XPG was acetylated in all samples, again indicating that DRB did not inhibit XPG acetylation (Figure 2C).

A

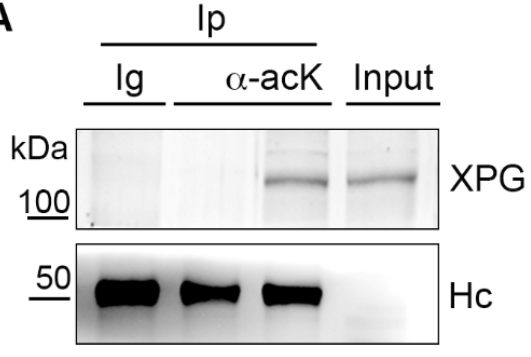

B

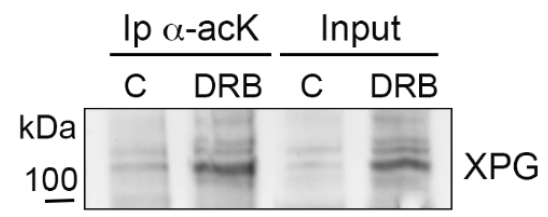

\section{C}

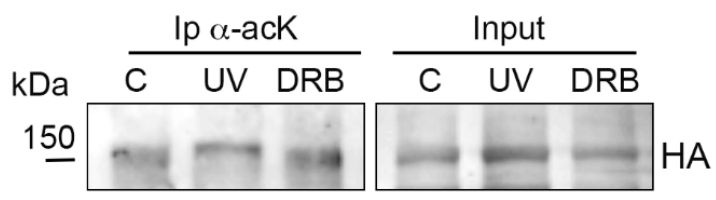

Figure 2. XPG acetylation is not affected by inhibition of transcription. (A) Specificity of immunoprecipitation (Ip) of acetylated XPG was tested in LF-1 cell extracts by incubation with purified immunoglobulin G (Ig), or with anti-acK antibody in the absence of HDAC inhibitors. Hc: Ig heavy chains. (B) Immunoprecipitation (Ip) of acetylated proteins with anti-acK antibody from LF-1 chromatin-bound nuclear extracts of (C) untreated or DRB-treated samples. Western blot analysis of XPG in input and Ip is shown. Input loading is 1/10 of total nuclear extracts. (C) Immunoprecipitation of acetylated proteins with anti acK antibody from chromatin-bound nuclear extracts of HEK293 cells expressing HA-tagged XPG. Untreated (C), UV-irradiated $\left(10 \mathrm{~J} / \mathrm{m}^{2}\right)$, and DRB-treated samples are shown. Western blot analysis of HA-tagged XPG in the input and Ip is shown. Input loading is $1 / 10$ of total nuclear extracts.

In order to establish the dependence of XPG chromatin association on its acetylation, immunoprecipitation with an anti-acK antibody was performed in $\mathrm{HaCaT}$ cells treated with C646, a specific inhibitor of p300/CBP [29]. The amount of acetylated XPG was significantly reduced in C646-treated cells (Figure 3A), as was the amount of PCNA, which is also acetylated by p300 and CBP [31]. Surprisingly, however, reduced XPG acetylation occurred in concomitance with an apparent accumulation of chromatin-bound XPG, observed in the input extract, as compared with untreated cells. To further understand whether acetylation could influence XPG chromatin association, CBP and p300 proteins were codepleted by RNA interference with siRNA in LF-1 fibroblasts, as previously described [27]. The efficiency of p300 and CBP depletion was further verified by immunofluorescence staining (Supplementary Figure S2). Western blot analysis of the chromatin-bound form of XPG in siRNA-treated LF-1 fibroblasts (Figure 3B) indicated that the depletion of both p300 and CBP induced an increase of more than two times in the level of chromatin-bound XPG after normalization to histone H3 content (Figure 3C). In addition, to investigate chromatin association at the microscopic level, we analyzed the nuclear distribution of XPG by immunofluorescence staining with a validated polyclonal antibody [32]. In LF-1 fibroblasts incubated with siRNA targeting p300 and CBP, the levels of chromatin-bound XPG showed a redistribution of XPG immunofluorescence from a fine punctuated uniform pattern, to clustered areas in a dark background (Figure 3D), with a concomitant increase in fluorescence intensity (Figure 3E). This redistribution was not observed in cells incubated with control siRNA, or targeting another protein, such as Cul4A (not shown). In order 
to confirm objectively the redistribution of XPG, texture analysis by using the gray level co-occurrence matrix (GLCM) was performed [33]. Results reported in Table 1 indicate that, in cells depleted of p300/CBP, the parameters of texture homogeneity (angular second moment, inversed difference moment, and correlation) were lower, while the parameter of disorganization (entropy) was higher, respectively, than the corresponding values measured in cells incubated with control siRNA oligos.

A

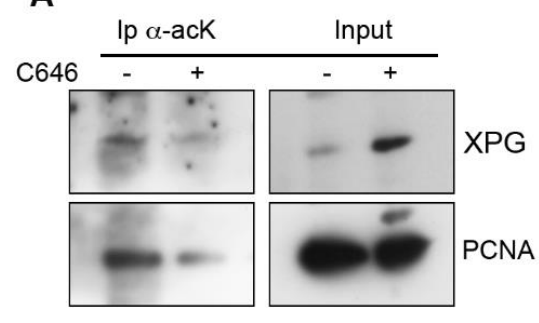

D

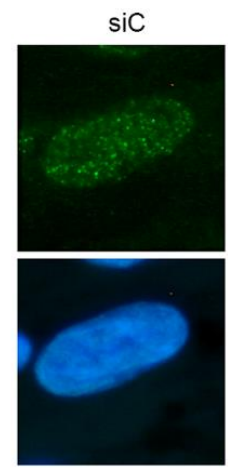

B

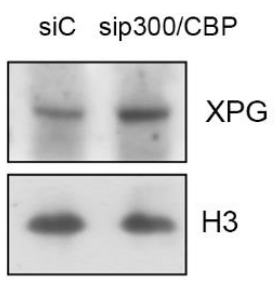

C

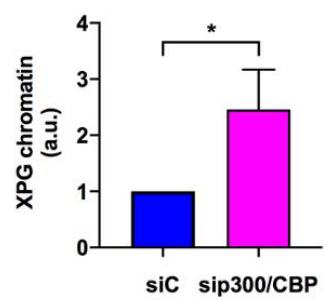

$E$

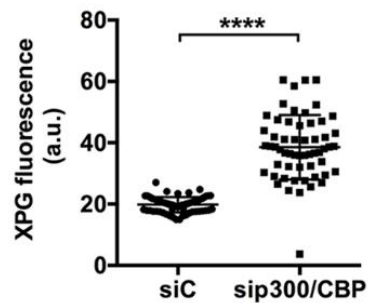

Figure 3. Inhibition of p300/CBP activity induces increase in and redistribution of chromatin-bound XPG. (A) Immunoprecipitation (Ip) of acetylated proteins from chromatin-bound nuclear extracts of (C) untreated or C646-treated LF-1 fibroblasts. Western blot analysis of XPG and PCNA in the input and Ip is shown. (B) Western blot analysis of XPG and histone H3 proteins in chromatin-bound nuclear extracts of LF-1 fibroblasts previously incubated with nontargeting siRNA (siC) or siRNA to p300 and CBP (sip300/CBP). (C) Densitometry quantification of XPG bands in the Western blots of chromatin-bound nuclear extracts of LF-1 fibroblasts previously incubated with nontargeting siRNA (siC) or siRNA to $\mathrm{p} 300$ and CBP (sip300/CBP). Densitometry values normalized to histone H3 content and given as fold value of the amount in siC samples. Mean values \pm S.D. of three experiments are shown. (* $p<0.05)$. (D) Immunofluorescence staining of chromatin-bound XPG (green fluorescence) and DNA (blue fluorescence) in LF-1 fibroblasts previously incubated with nontargeting siRNA (siC) or siRNA to p300 and CBP (sip300/CBP). Scale bar $=10 \mu \mathrm{m}$. (E) Quantification of XPG fluorescence intensity in LF-1 fibroblasts prepared as in (D). Fluorescence was quantified by Image J as described in Materials and Methods. At least 50 cells (from two independent experiments) were analyzed. Error bars show SEM $\left({ }^{* * * *} p<0.0001\right)$.

\subsection{Inhibition of Transcription Induces XPG Accumulation and Relocation}

As both p300 and CBP are involved in basal transcription [34], the relocation of XPG could not be unequivocally attributed to a reduction in its acetylation, but could be the result of transcription impairment. In fact, C646 was also able to inhibit basal transcription as determined by BrU incorporation (Supplementary Figure S3). On the basis of these results, we sought to understand whether blocking RNA synthesis could also influence the extent and nuclear distribution of chromatin-bound XPG. To this end, LF-1 cells were treated with the RNA polymerase II (pol II) inhibitors AD or DRB, or with the topo I inhibitor CPT, in addition to C646. As compared with untreated control cells, 
all these compounds induced a significant increase in the amount of chromatin-bound XPG, as determined by Western blot analysis (Figure 4A). The quantification of band intensities by densitometry and normalization to histone $\mathrm{H} 3$ indicated that the levels of chromatin-bound XPG increased by 2-3 times, as compared with in the untreated control cells (Figure 4B). In concomitance, redistribution of XPG to nuclear clusters similar to those observed after depletion of p300/CBP was found in C646-treated cells (Figure 4C), and with all other transcription inhibitors (Table 2), including RNA pol I inhibitor BMH21 (Supplementary Figure S4). Similar clustered localization was observed for XPF, which is the other endonuclease working in concert with XPG in both NER and for R-loop processing (Figure 4D). However, the redistribution of XPG was not a general phenomenon involving nuclear proteins, since PCNA did not show similar compartmentalization (Supplementary Figure S5A).

Table 1. Texture analysis of XPG immunofluorescence distribution after siRNA-mediated depletion of p300 and CBP.

\begin{tabular}{|c|c|c|c|c|}
\hline \multirow[b]{2}{*}{ GLCM Feature } & \multirow[b]{2}{*}{ Sample } & \multicolumn{3}{|c|}{ Distance } \\
\hline & & $d=3$ & $d=6$ & $\mathrm{~d}=9$ \\
\hline \multirow{2}{*}{$\begin{array}{l}\text { Angular second } \\
\text { moment }\end{array}$} & siControl & $0.02100 \pm 0.001474$ & $0.01375 \pm 0.001066$ & $0.01073 \pm 0.0008634$ \\
\hline & $\operatorname{sip} 300 / C B P$ & $0.003390 \pm 0.0009269 * * * *$ & $0.002188 \pm 0.0006927^{* * * *}$ & $0.001645 \pm 0.0005484^{* * * *}$ \\
\hline \multirow{2}{*}{ Correlation } & siControl & $0.03145 \pm 0.002600$ & $0.02413 \pm 0.001858$ & $0.01782 \pm 0.001343$ \\
\hline & sip300/CBP & $0.0004503 \pm 1.790 \times 10^{-5 * * * *}$ & $0.0004200 \pm 1.565 \times 10^{-5 * * * *}$ & $0.0003847 \pm 1.319 \times 10^{-5 * * * *}$ \\
\hline \multirow{2}{*}{$\begin{array}{l}\text { Inverse difference } \\
\text { moment }\end{array}$} & siControl & $0.5568 \pm 0.01724$ & $0.4205 \pm 0.01650$ & $0.3492 \pm 0.01563$ \\
\hline & $\operatorname{sip} 300 / C B P$ & $0.2367 \pm 0.01459^{* * * *}$ & $0.1446 \pm 0.01191^{* * * *}$ & $0.1036 \pm 0.009709^{* * * *}$ \\
\hline \multirow{2}{*}{ Entropy } & siControl & $4.839 \pm 0.1121$ & $5.331 \pm 0.1188$ & $5.566 \pm 0.1207$ \\
\hline & $\operatorname{sip} 300 / C B P$ & $7.364 \pm 0.1223$ **** & $7.909 \pm 0.1215^{* * * *}$ & $8.171 \pm 0.1185^{* * * *}$ \\
\hline
\end{tabular}

Distance in pixel (d): steps used for the GLCM analysis. ${ }^{* * * *} p<0.0001$.

Table 2. Texture analysis of XPG immunofluorescence distribution after inhibition of transcription with DRB or C646.

\begin{tabular}{|c|c|c|c|c|}
\hline & & & Distance & \\
\hline GLCM Feature & Sample & $d=3$ & $d=6$ & $\mathrm{~d}=9$ \\
\hline \multirow{3}{*}{$\begin{array}{l}\text { Angular second } \\
\text { moment }\end{array}$} & Control & $0.01605 \pm 0.0009294$ & $0.009992 \pm 0.0006226$ & $0.007657 \pm 0.0004850$ \\
\hline & DRB & $0.01261 \pm 0.0007157^{* *}$ & $0.007838 \pm 0.0005743 *$ & $0.006063 \pm 0.0004919$ * \\
\hline & $\mathrm{C} 646$ & $0.01288 \pm 0.0006540 * *$ & $0.007909 \pm 0.0004422^{* *}$ & $0.006070 \pm 0.0003523^{* *}$ \\
\hline \multirow{3}{*}{ Correlation } & Control & $0.03624 \pm 0.002843$ & $0.02972 \pm 0.002082$ & $0.02411 \pm 0.001495$ \\
\hline & DRB & $0.02652 \pm 0.001212 * * *$ & $0.02175 \pm 0.0009079^{* * *}$ & $0.01731 \pm 0.0006764^{* * * *}$ \\
\hline & C646 & $0.01612 \pm 0.0009309^{* * * *}$ & $0.01340 \pm 0.0007202^{* * * *}$ & $0.01073 \pm 0.0005686^{* * * *}$ \\
\hline \multirow{3}{*}{$\begin{array}{l}\text { Inverse difference } \\
\text { moment }\end{array}$} & Control & $0.6188 \pm 0.007871$ & $0.4613 \pm 0.008658$ & $0.3737 \pm 0.008313$ \\
\hline & DRB & $0.5411 \pm 0.009005^{* * * *}$ & $0.3875 \pm 0.008952^{* * * *}$ & $0.3106 \pm 0.008126^{* * * *}$ \\
\hline & C646 & $0.5361 \pm 0.009026^{* * * *}$ & $0.3875 \pm 0.009044^{* * * *}$ & $0.3115 \pm 0.008228^{* * * *}$ \\
\hline \multirow{3}{*}{ Entropy } & Control & $4.561 \pm 0.04921$ & $5.049 \pm 0.05104$ & $5.309 \pm 0.05207$ \\
\hline & DRB & $4.953 \pm 0.03865^{* * * *}$ & $5.463 \pm 0.03992^{* * * *}$ & $5.719 \pm 0.03912^{* * * *}$ \\
\hline & C646 & $5.041 \pm 0.04941^{* * * *}$ & $5.541 \pm 0.05167^{* * * *}$ & $5.794 \pm 0.05214^{* * * *}$ \\
\hline
\end{tabular}

Distance in pixel (d): steps used for the GLCM analysis. ${ }^{*} p<0.05 ;{ }^{* *} p<0.01 ;{ }^{* * *} p<0.001 ;{ }^{* * * *} p<0.0001$.

XPG relocation was observed in both LF-1 fibroblasts and HaCaT keratinocytes (Supplementary Figure S5B), and the quantification of cells showing this pattern indicated that the phenomenon occurred independently of cell type (Figure 4E). Since transcription inhibition by DRB is known to be reversible, we also investigated whether XPG focus formation followed a similar fate. Interestingly, the removal of DRB or C646 from the medium resulted in a significant reduction in cells with XPG clusters, as seen at $18 \mathrm{~h}$ after the end of treatment (Figure 4F). Transcription inhibition induces the segregation to perinucleolar sites of a number of transcription-related proteins, including CDK2 [35]. Therefore, we investigated whether XPG relocation could overlap the nuclear distribution of this factor. 
However, after DRB treatment, the relocation of CDK2 appeared to be markedly different from that of XPG (Supplementary Figure S6).

A

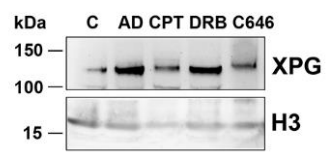

C

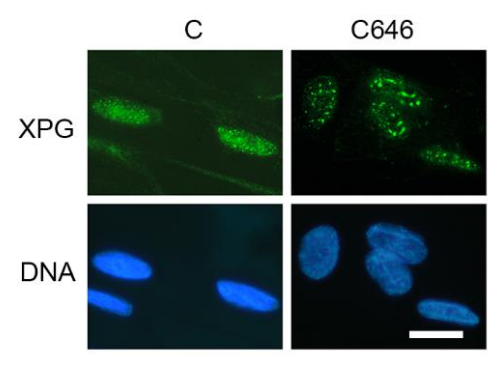

E

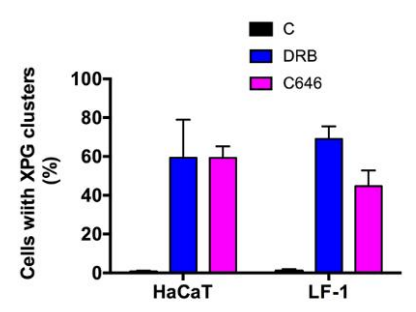

B

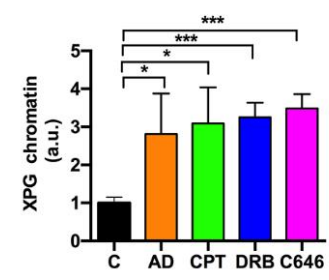

D

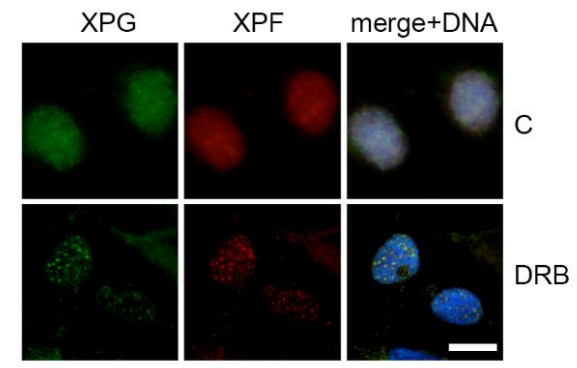

$\mathbf{F}$

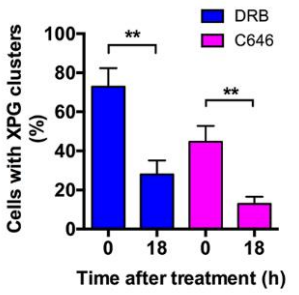

Figure 4. Accumulation and nuclear localization of XPG after transcription inhibition. (A) Western blot analysis of chromatin-bound fraction of XPG in LF-1 fibroblasts after treatment with indicated transcription inhibitors, as described in Materials and Methods; histone $\mathrm{H} 3$ is shown as a loading control. (B) Quantitative densitometric analysis of chromatin-bound fraction of XPG in LF-1 fibroblasts. Mean values \pm S.D. of at least three different experiments shown. ${ }^{*} p<0.05 ;{ }^{* *} p<0.01 ;{ }^{* * *} p<0.001$. (C) Immunofluorescence of chromatin-bound XPG in LF-1 fibroblasts in (C) untreated and C646treated (C646) samples. Scale bar $=10 \mu \mathrm{m}$. (D) Immunofluorescence analysis of chromatin-bound XPG (green fluorescence) and XPF (red fluorescence) in HeLa cells in untreated (C) and DRB-treated (DRB) samples. Scale bar $=10 \mu \mathrm{m}$. (E) Quantification of cells showing clustered distribution of XPG in $\mathrm{HaCaT}$ and in LF-1 untreated culture samples (C), or after treatment with DRB, or C646. Mean values \pm S.D. of three experiments shown. (F) Quantification of cells showing clustered distribution of XPG in LF-1 fibroblasts at end of the treatment $(0 \mathrm{~h})$, and after $18 \mathrm{~h}$ recovery in the absence of the inhibitor. Mean values \pm S.D. of three experiments shown. $\left({ }^{* *} p<0.01\right)$.

\subsection{XPG Relocation Occurs in Concomitance with Histone $\gamma-H 2 A X$ and $R$-Loop Formation}

The inhibition of transcription by AD or CPT induces DNA damage and the phosphorylation of histone H2AX ( $\gamma$-H2AX) [36,37]. Therefore, to understand whether XPG relocation was related to a similar DNA damage response, we analyzed the presence of $\gamma-\mathrm{H} 2 \mathrm{AX}$ positive cells in LF-1 fibroblasts treated with DRB or C646. The quantification of immunofluorescence and the percentage of positive cells suggested that C646 was able to trigger the appearance of $\gamma-\mathrm{H} 2 \mathrm{AX}$ better than DRB could, whose signal was, however, significantly higher when compared with that of untreated control cells (Figure 5A,B and Supplementary S7). Next, we investigated whether this response was induced by the 
formation of R-loops, since XPG is involved in the processing of such structures [18]. Immunostaining with antibody S9.6 was performed in cells treated with DRB or with C646. Results indicated that both drugs induced the appearance of cells positive to S9.6 immunofluorescence staining (Figure 5C,D and Supplementary Figure S7). To understand whether the relocation of XPG occurred in dependence of R-loop formation, their colocalization by double immunofluorescence staining could not be performed due to differences in the optimal fixation procedure for each antigen. Therefore, we analyzed the proximity of XPG to R-loops using PLA, which provides better resolution than that of confocal microscopy. Results indicated that XPG colocalized with R-loops with a higher frequency after C646 than that after DRB treatment (Figure 5E,F).

A

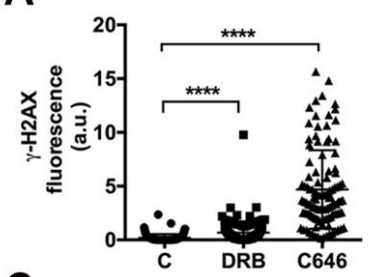

C

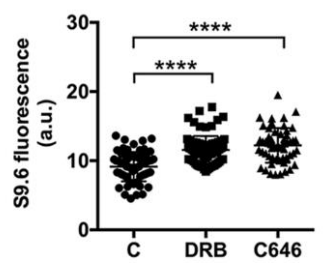

B

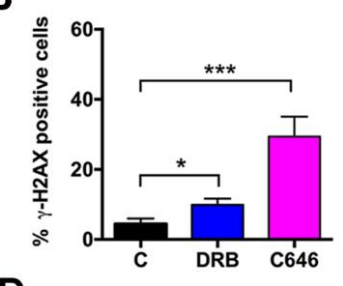

D

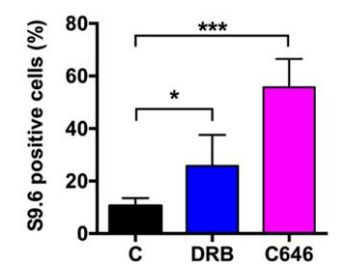

$E$

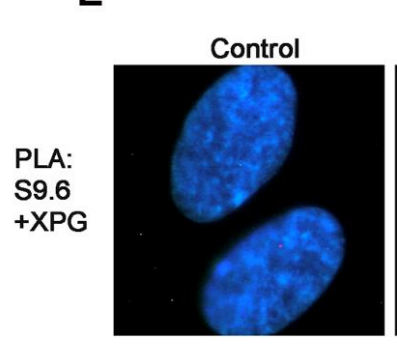

$\mathbf{F}$

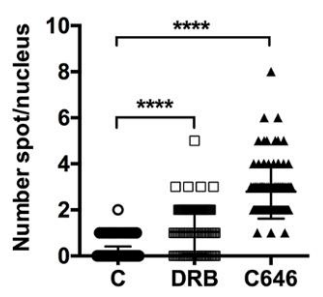

Figure 5. Relocalization of XPG induced by transcription inhibition occurs in concomitance with the phosphorylation of histone H2AX and R-loop formation. (A) Quantitative analysis of histone $\gamma-\mathrm{H} 2 \mathrm{AX}$ immunofluorescence staining in LF-1 fibroblasts treated with DRB or C646. At least 50 cells (from 2-3 independent experiments) were measured. Error bars with SEM shown. $(* * * *<<0.0001)$ (B) Quantitative analysis of positivity to immunofluorescence staining of histone $\gamma$-H2AX, in LF-1 fibroblasts treated with indicated inhibitors of transcription. Mean values \pm S.D. of three experiments shown. ( ${ }^{*} p<0.05$; ${ }^{* * *} p<0.001$ ). (C) Quantitative analysis of S9.6 immunofluorescence staining in LF-1 fibroblasts treated with indicated inhibitors. At least 50 cells (from 2-3 independent experiments) measured. Error bars with SEM shown. (*** $p<0.0001$ ). (D) Quantitative analysis of positivity to immunofluorescence staining of RNA/DNA hybrids with S9.6 antibody, in LF-1 fibroblasts treated with indicated inhibitors. Mean values \pm S.D. of three experiments shown. ${ }^{*} p<0.05$; $* * * p<0.001$ ). (E) PLA assay performed in LF-1 fibroblasts after treatment with C646. Red spots represent the positivity of the reaction. Scale bar: $10 \mu \mathrm{m}$. (F) Quantitative analysis of the number of PLA spots/nucleus in (C) untreated, DRB-, or C646-treated LF-1 fibroblasts. Scatter plot shows pooled data from two independent experiments. Number of spots in at least 50 cells/experiment was counted. $\left.{ }^{* * * *} p<0.0001\right)$. 


\section{Discussion}

In this study, we investigated the chromatin association of XPG during normal cell growth and after the inhibition of transcription, revealing a novel feature of XPG nuclear localization. Our results showed that this protein is accumulated at nuclear clusters when transcription is impaired both by RNA pol II and topo I inhibitors. However, this accumulation was also observed after the inhibition of p300 and CBP either with C646 or by siRNA-mediated depletion of both KAT enzymes whose activity is involved in XPG acetylation [27]. Since p300 and CBP play enzymatic and nonenzymatic functions during transcription [34], our results suggest that XPG was accumulated due to transcription inhibition, and not (at least not only) because of reduced acetylation. XPG remained acetylated after treatment with DRB, thus suggesting that the redistribution of XPG as nuclear clusters was not specifically related to XPG acetylation.

Some nuclear proteins involved in transcription may undergo redistribution by forming nucleolar caps after treatment with AD or DRB [35]. Although a similarity was observed with XPG relocation, the process appears to be different, since we did not find correlation of localization between XPG and CDK2, one of the factors observed to form these caps during transcription inhibition [35]. In this experiment, we had to use an anti-XPG monoclonal antibody (instead of the polyclonal), to assess the colocalization of XPG and CDK2. Therefore, a difference in epitope binding might have influenced these results, and further analysis is required.

Our results showed that XPG accumulated in regions in which XPF was also present, suggesting that relocation was related to a function involving both endonucleases, such as the processing of R-loops [18]. In fact, both DRB and C646 induced the formation of RNA/DNA hybrids, as detected by S9.6 immunofluorescence. PLA confirmed that the inhibition of transcription induced XPG redistribution at the same sites in which R-loops were detected. The evidence that, in our experimental conditions, the formation of R-loops (and of $\gamma-\mathrm{H} 2 \mathrm{AX}$ ) was higher with C646 than that with DRB treatment may be explained by their different mechanisms $[29,38]$. DRB may have blocked RNA synthesis, thereby reducing the formation of new R-loops during the treatment period. In contrast, with C646 being an inhibitor of p300/CBP, it may have impaired RNA synthesis with an indirect mechanism while still allowing for the formation and accumulation of R-loops. XPG accumulation was induced by both drugs, suggesting that the recruitment of XPG was triggered by a mechanism sensing the inhibition of RNA synthesis rather than the R-loop structure itself. Further studies would clarify this aspect.

In conclusion, our results showed that XPG is relocated to nuclear clusters after transcriptional stress, suggesting that this new distribution reflects the processing of $\mathrm{R}$ loops.

\section{Materials and Methods}

\subsection{Cell Cultures and Treatments}

LF-1 human normal embryonic fibroblasts (from J. Sedivy, Brown University, Providence, RI, USA) were grown in MEM supplemented with 10\% fetal bovine serum (FBS), streptomycin $(100 \mu \mathrm{g} / \mathrm{mL}) /$ penicillin (100 IU), and $1 \mathrm{mM}$ glutamine. Human immortalized keratinocyte cell line HaCaT (IZLER, BS, Italy) was grown in DMEM high-glucose medium supplemented with 10\% FBS, streptomycin $(200 \mu \mathrm{g} / \mathrm{mL}) /$ penicillin $(200 \mathrm{IU})$, and 2 mM glutamine. Human epithelial kidney 293 (HEK 293) and HeLa cells were grown in DMEM high glucose as above. HaCaT and LF-1 cells were treated with $20 \mu \mathrm{M}$ C646 (Selleck Chem., Houston, TX, USA) in serum-free medium for $2 \mathrm{~h}$ at $37^{\circ} \mathrm{C}$. Then, the serum was added to the medium, and the treatment continued for $4 \mathrm{~h}$. 5,6-Dichloro-1$\beta$-D-ribofuranosylbenzimidazole (DRB), camptothecin (CPT), and actinomycin D (AD) were obtained from Sigma Aldrich (Milano, Italy). DRB was used at $100 \mu \mathrm{M}$ for HaCaT cells and $50 \mu \mathrm{M}$ for LF-1 cells in complete medium for $6 \mathrm{~h}$ at $37^{\circ} \mathrm{C}$. Treatments with CPT $(5 \mu \mathrm{M})$ or $\mathrm{AD}(10 \mu \mathrm{g} / \mathrm{mL})$ were also performed in complete medium for $6 \mathrm{~h}$ at $37^{\circ} \mathrm{C}$. RNA pol I inhibitor BMH21 [39], obtained from TargetMol (Boston, MA, USA), was used at the 
final concentration of $2 \mu \mathrm{M}$. In some experiments, cells were exposed to UV-C irradiation $\left(10 \mathrm{~J} / \mathrm{m}^{2}\right)$ with a TUV-9 lamp (Philips) and collected $30 \mathrm{~min}$ later [27,31].

Small-interfering (si) RNA oligos for p300 and CBP (ON-TARGET PLUS smart pool) were obtained from Dharmacon (Horizon Discovery, Cambridge, U.K.). Depletion of p300 and CBP was performed by incubating LF-1 fibroblasts for $72 \mathrm{~h}$ with oligos ( $20 \mathrm{nM}$ each) to p300 and CBP, using INTERFERin (Polyplus, Illkirch, France) as transfection reagent. Nontargeting siRNA (Dharmacon) was used as control.

\subsection{Flow Cytometry}

HaCaT cells were harvested with trypsin, transferred to $15 \mathrm{~mL}$ tubes, centrifuged for $5 \mathrm{~min}$ at $300 \times g$ at RT, and washed with PBS. Samples were fixed with $0.5 \%$ formaldehyde in PBS for $5 \mathrm{~min}$ at RT, then centrifuged and resuspended in physiological saline solution to which ethanol was added to a final $70 \%$ concentration for storing at $-20{ }^{\circ} \mathrm{C}$. After thawing, cells were washed in PBS and incubated in blocking solution (1\% BSA in PBT) for $30 \mathrm{~min}$. Cells were then incubated at RT for $1 \mathrm{~h}$ with anti-XPG polyclonal antibody (Sigma, 1:200), followed by three washes in 1\% BSA in PBS and incubation for $30 \mathrm{~min}$ at RT with secondary Alexa 488-conjugated antirabbit antibody (1:200). At the end, cells were again washed 3 times in PBT and incubated overnight a $4{ }^{\circ} \mathrm{C}$ in a solution containing $50 \mu \mathrm{g} / \mathrm{mL}$ propidium iodide (PI) and $0.5 \mathrm{mg} / \mathrm{mL}$ RNase A (Sigma) in PBS. Fluorescence signals were measured using a Partec PAS II flow cytometer (Partec, Münster, Germany).

\subsection{Western Blot and Immunoprecipitation}

Protein extraction was performed with a hypotonic buffer $\left(1 \mathrm{~mL} / 10^{7}\right.$ cells $)$ containing $10 \mathrm{mM}$ Tris- $\mathrm{HCl}$ ( $\mathrm{pH} 8.0), 2.5 \mathrm{mM} \mathrm{MgCl}, 10 \mathrm{mM} \mathrm{Na} \beta$-glycerophosphate, $0.5 \%$ Igepal, $1 \mathrm{mM}$ PMSF, $1 \mathrm{mM}$ DTT, $1 \mathrm{mM} \mathrm{Na} \mathrm{VO}_{4}, 10 \mathrm{mM} \mathrm{Na}$ butyrate, $200 \mathrm{ng} / \mathrm{mL}$ TSA (Sigma), protease and phosphatase inhibitor cocktails (Sigma), for $8 \mathrm{~min}$ in ice. The detergent-soluble fraction was taken apart, and after two washes in isotonic buffer containing $10 \mathrm{mM}$ Tris- $\mathrm{HCl}$ ( $\mathrm{pH} 8.0), 150 \mathrm{mM} \mathrm{NaCl}$, and inhibitors as above, nuclear pellets were incubated for $20 \mathrm{~min}$ at $4{ }^{\circ} \mathrm{C}$ with $20 \mathrm{U} / 10^{6}$ cells of DNase I (Sigma) in half volume of initial lysis, in $10 \mathrm{mM}$ Tris$\mathrm{HCl}$ buffer (pH 8.0), $2.5 \mathrm{mM} \mathrm{MgCl}_{2}$ and $20 \mathrm{mM} \mathrm{NaCl}$, as described [27,30]. At the end of the reaction, the total volume was mixed with loading buffer. Samples were run on NuPAGE $4-12 \%$ gels (ThermoFisher) and transferred to nitrocellulose for Western blot analysis. Membranes were blocked in 5\% BSA in PBS containing 0.2\% Tween 20, then incubated for 1 $\mathrm{h}$ in primary antibody (listed in Table S1) solution in blocking buffer. After several washes in the same buffer, and incubation with relevant HRP-conjugated secondary antibody, the reaction was developed with chemiluminescence substrates (Cyanagen, Bologna, Italy), and signals were detected with a Westar R Imager (HiTech Cyanagen, Bologna, Italy).

For immunoprecipitation, the chromatin-bound fraction was diluted in isotonic buffer containing inhibitor cocktails as above, immunoprecipitated with antiacetyl lysine (acK) mouse monoclonal antibody (clone 4G12, Millipore), or with rabbit polyclonal antibody anti-CBP (Santa Cruz Biotech., St. Cruz, CA, USA), previously coupled to protein Gmagnetic beads (Dynabeads, ThermoFisher, Monza, Italy) and incubated at $4{ }^{\circ} \mathrm{C}$,for $3 \mathrm{~h}$ on a rotating wheel. Immunocomplexes were isolated and washed three times in the same buffer, mixed with loading buffer, and analyzed by Western blot with anti-XPG or other relevant antibodies (Table S1). The specificity of the anti-acK antibody (31) was tested by incubating cell extracts in the absence of HDAC inhibitors Na butyrate and TSA. Another control consisted of purified mouse IgG (Sigma) in place of the anti-acK antibody.

\subsection{Immunofluorescence}

Immunofluorescence detection of chromatin-bound XPG was performed in LF-1 fibroblasts) or $\mathrm{HaCaT}$ cells lysed in situ with hypotonic buffer containing $10 \mathrm{mM}$ Tris- $\mathrm{HCl}$ ( $\mathrm{pH}$ 8.0), $3 \mathrm{mM} \mathrm{MgCl}$, $10 \mathrm{mM}$ Na $\beta$-glycerophosphate, $0.1 \%$ Igepal, $0.2 \mathrm{mM}$ PMSF, $0.1 \mathrm{mM}$ $\mathrm{Na}_{3} \mathrm{VO}_{4}$, and protease inhibitors, for $8 \mathrm{~min}$ at $4{ }^{\circ} \mathrm{C}$ [27]. Cells washed in the same buffer without detergent and then fixed in $2 \%$ formaldehyde in PBS followed by permeabilization 
in $70 \%$ ethanol. After saturation with 1\% BSA-0.2 Tween 20, cells were incubated for $1 \mathrm{~h}$ with anti-XPG polyclonal antibody (Sigma) (diluted 1:200), and anti-PCNA PC10 monoclonal antibody (Dako, Agilent, Milano, Italy) diluted 1:100. Secondary antimouse and antirabbit antibodies labeled with Alexa 594 (red fluorescence), or Dylight 488 (green fluorescence), respectively, were used. DNA was stained with Hoechst 33258.

Histone $\gamma-\mathrm{H} 2 \mathrm{AX}$ detection was performed after fixation of whole cells with $2 \%$ formaldehyde in PBS followed by permeabilization in 70\% ethanol, and blocking step as above. The primary antibody to $\gamma$-H2AX (clone JBW301) was diluted 1:3000, followed by the secondary antimouse antibody labeled with Alexa 488.

For R-loop detection, after treatment with DRB, C646 or CPT, cells were fixed in cold $100 \%$ methanol and stored for $15 \mathrm{~min}$ at $-20{ }^{\circ} \mathrm{C}$. After washing in PBS, cells were incubated with blocking solution (3\% BSA, 0.1\% Tween 20,4xSSC) for $30 \mathrm{~min}$ at RT. Slides were washed for 5 min with PBT solution (PBS $+0.1 \%$ Tween 20) and incubated overnight at $4{ }^{\circ} \mathrm{C}$ with $50 \mu \mathrm{L}$ of blocking solution containing the S9.6 primary antibody (1:1000). Subsequently, cells were washed three times for 10 min each with PBT and then incubated 30 min with $50 \mu \mathrm{L}$ of solution containing $4 \times$ SSC buffer and the secondary antibody, goat antimouse Alexa-488, conjugated (1:200). To improve visualization, a subsequent step of amplification (30 $\mathrm{min}$ ) was performed with donkey-antigoat antibody Alexa-488 conjugated (1:300). After that, slides were washed three times for 10 min each with PBT, then incubated 5 min with a solution of Hoechst 33,258 dye $(0.1 \mu \mathrm{g} / \mathrm{mL})$ in PBS; after two washes in PBS, slides were mounted in Mowiol. Samples were observed with an Olympus BX51 fluorescence microscope using 100x oil immersion, and pictures were captured by an Olympus C4040 digital camera. Fluorescence intensity was analyzed with Image J software (version 1.52a, NIH, Bethesda, MA, USA), using DNA images to identify nuclei, and a particle-analysis tool, as described [40]. Analysis of XPG fluorescence redistribution was performed through the gray level co-occurrence matrix (GLCM) plugin of Image J [33]. Only nuclei with at least two clearly identifiable clusters were considered for this analysis.

\subsection{Proximity Ligation Assay}

Proximity ligation assay (PLA) was performed using the Duolink ${ }^{\circledR}$ PLA kit (Sigma) following the provided protocol. LF-1 cells seeded on coverslips were treated with C646 or DRB, as indicated above and then fixed with formaldehyde $(2 \%)$ solution in PBS, followed by cold ethanol $(70 \%)$ permeabilization. Each coverslip was incubated in a humidity chamber $\left(1 \mathrm{~h}\right.$ at $\left.37^{\circ} \mathrm{C}\right)$ with Duolink ${ }^{\circledR}$ blocking solution, and then with $50 \mu \mathrm{L}$ of Duolink ${ }^{\circledR}$ antibody diluent containing the two primary antibodies: anti-XPG (polyclonal, St John's Laboratory), and anti-RNA/DNA hybrid monoclonal S9.6, both diluted 1:100. After that, coverslips were washed two times (5 min each) with buffer A at RT, and incubated in a preheated humidity chamber for $1 \mathrm{~h}$ at $37^{\circ} \mathrm{C}$ with PLUS and MINUS PLA probes diluted in Duolink ${ }^{\circledR}$ antibody diluent. Then, after two further washings ( 5 min each), samples were incubated in a preheated humidity chamber for $30 \mathrm{~min}$ at $37^{\circ} \mathrm{C}$ with Ligase diluted in the Duolink $^{\circledR}$ ligation buffer. Once the ligation step had been completed, slides were washed two times ( 5 min each) with buffer A at RT, and the amplification step was performed in a preheated humidity chamber for $100 \mathrm{~min}$ at $37^{\circ} \mathrm{C}$ with polymerase diluted in the Duolink ${ }^{\circledR}$ amplification buffer containing red-fluorescence-labeled nucleotides for the rolling-circle amplification. Coverslips were washed two times for $10 \mathrm{~min}$ each with buffer B at RT. Lastly, DNA was stained using Hoechst 33,258 $(0.1 \mu \mathrm{g} / \mathrm{mL}$ in PBS) for $2 \mathrm{~min}$. After two washes in PBS, coverslips were mounted on slides in Mowiol and observed as described above.

\subsection{Statistical Analysis}

At least three biological replicates (unless otherwise stated) were performed for each experiment. Statistical analysis was performed with Prism 6 software (GraphPad, San Diego, CA, USA) used to calculate significance with the Student $t$ test (two-tailed), with $p$ values $<0.05$ considered to be significant. 
Supplementary Materials: The following are available online at https://www.mdpi.com/article/10 $.3390 /$ ijms22126589/s1.

Author Contributions: C.S. and G.T. contributed equally to this work. Conceptualization, E.P. and I.D.; methodology, E.P., C.S., and G.T.; investigation, I.D., C.S., G.T., O.C., L.A.S., and E.P.; data curation, C.S. and G.T..; image analysis, G.T.; writing-original-draft preparation, E.P.; writingreview and editing, E.P., I.D., C.S., L.A.S., and O.C.; funding acquisition, E.P. All authors have read and agreed to the published version of the manuscript.

Funding: This research was funded by Italian Association for Cancer Research (AIRC), grant number IG 17041, to E.P., and in part by a grant to L.A.S. from the Italian Ministry of Education, University, and Research (MIUR), under the "Dipartimenti di Eccellenza" (2018-2022) initiative.

Institutional Review Board Statement: Not applicable.

Informed Consent Statement: Not applicable.

Data Availability Statement: Data presented in this study are available upon reasonable request to the corresponding author.

Acknowledgments: We wish to thank J.M. Sedivy (Brown University, Providence, RI, USA) for providing LF-1 fibroblasts, J.M. Egly (IGBMC, Strasbourg, France) for providing the HA-XPG plasmid, and S. Sabbioneda (IGM-CNR, Italy) for providing the S9.6 antibody.

Conflicts of Interest: The authors declare no conflict of interest. The funders had no role in the design of the study; in the collection, analyses, or interpretation of data; in the writing of the manuscript, or in the decision to publish the results.

\section{References}

1. Clarkson, S. The XPG story. Biochimie 2003, 85, 1113-1121. [CrossRef]

2. Schärer, O.D. XPG: Its products and biological roles. Adv. Exp. Med. Biol. 2009, 637, 83-92. [CrossRef]

3. O'Donovan, A.; Davies, A.A.; Moggs, J.G.; West, S.; Wood, R.D. XPG endonuclease makes the $3^{\prime}$ incision in human DNA nucleotide excision repair. Nat. Cell Biol. 1994, 371, 432-435. [CrossRef]

4. Evans, E.; Fellows, J.; Coffer, A.; Wood, R.D. Open complex formation around a lesion during nucleotide excision repair provides a structure for cleavage by human XPG protein. EMBO J. 1997, 16, 625-638. [CrossRef]

5. Staresincic, L.; Fagbemi, A.F.; Enzlin, J.H.; Gourdin, A.M.; Wijgers, N.; Dunand-Sauthier, I.; Giglia-Mari, G.; Clarkson, S.G.; Vermeulen, W.; Schärer, O.D. Coordination of dual incision and repair synthesis in human nucleotide excision repair. $E M B O J$. 2009, 28, 1111-1120. [CrossRef]

6. Fagbemi, A.F.; Orelli, B.; Schärer, O.D. Regulation of endonuclease activity in human nucleotide excision repair. DNA Repair 2011, 10, 722-729. [CrossRef]

7. Marteijn, J.; Lans, H.; Vermeulen, W.; Hoeijmakers, J.H.J. Understanding nucleotide excision repair and its roles in cancer and ageing. Nat. Rev. Mol. Cell Biol. 2014, 15, 465-481. [CrossRef]

8. Lehmann, A.R. DNA repair-deficient diseases, xeroderma pigmentosum, Cockayne syndrome and trichothiodystrophy. Biochimie 2003, 85, 1101-1111. [CrossRef] [PubMed]

9. Nouspikel, T. Nucleotide excision repair and neurological diseases. DNA Repair 2008, 7, 1155-1167. [CrossRef]

10. Niedernhofer, L.J.; Bohr, V.A.; Sander, M.; Kraemer, K.H. Xeroderma pigmentosum and other diseases of human premature aging and DNA repair: Molecules to patients. Mech. Ageing Dev. 2011, 132, 340-347. [CrossRef]

11. Lee, S.-K.; Yu, S.-L.; Prakash, L.; Prakash, S. Requirement of Yeast RAD2, a Homolog of Human XPG Gene, for Efficient RNA Polymerase II Transcription: Implications for Cockayne Syndrome. Cell 2002, 109, 823-834. [CrossRef]

12. Ito, S.; Kuraoka, I.; Chymkowitch, P.; Compe, E.; Takedachi, A.; Ishigami, C.; Coin, F.; Egly, J.-M.; Tanaka, K. XPG Stabilizes TFIIH, Allowing Transactivation of Nuclear Receptors: Implications for Cockayne Syndrome in XP-G/CS Patients. Mol. Cell 2007, 26, 231-243. [CrossRef]

13. Le May, N.; Mota-Fernandes, D.; Velez-Cruz, R.; Iltis, I.; Biard, D.; Egly, J.M. NER Factors Are Recruited to Active Promoters and Facilitate Chromatin Modification for Transcription in the Absence of Exogenous Genotoxic Attack. Mol. Cell 2010, 38, 54-66. [CrossRef]

14. Le May, N.; Fradin, D.; Iltis, I.; Bougnères, P.; Egly, J.-M. XPG and XPF Endonucleases Trigger Chromatin Looping and DNA Demethylation for Accurate Expression of Activated Genes. Mol. Cell 2012, 47, 622-632. [CrossRef]

15. Trego, K.S.; Chernikova, S.B.; Davalos, A.R.; Perry, J.J.P.; Finger, L.D.; Ng, C.; Tsai, M.-S.; Yannone, S.M.; Tainer, J.A.; Campisi, J.; et al. The DNA repair endonuclease XPG interacts directly and functionally with the WRN helicase defective in Werner syndrome. Cell Cycle 2011, 10, 1998-2007. [CrossRef] 
16. Trego, K.S.; Groesser, T.; Davalos, A.R.; Parplys, A.C.; Zhao, W.; Nelson, M.R.; Hlaing, A.; Shih, B.; Rydberg, B.; Pluth, J.M.; et al. Non-catalytic roles for XPG with BRCA1 and BRCA2 in homologous recombination and genome stability. Mol. Cell 2016, 61, 535-546. [CrossRef] [PubMed]

17. Wickramasinghe, V.O.; Venkitaraman, A.R. RNA Processing and Genome Stability: Cause and Consequence. Mol. Cell 2016, 61, 496-505. [CrossRef]

18. Sollier, J.; Stork, C.T.; García-Rubio, M.L.; Paulsen, R.D.; Aguilera, A.; Cimprich, K.A. Transcription-Coupled Nucleotide Excision Repair Factors Promote R-Loop-Induced Genome Instability. Mol. Cell 2014, 56, 777-785. [CrossRef]

19. Cristini, A.; Ricci, G.; Britton, S.; Salimbeni, S.; Huang, S.-Y.N.; Marinello, J.; Calsou, P.; Pommier, Y.; Favre, G.; Capranico, G.; et al. Dual Processing of R-Loops and Topoisomerase I Induces Transcription-Dependent DNA Double-Strand Breaks. Cell Rep. 2019, 28, 3167-3181.e6. [CrossRef]

20. Yasuhara, T.; Kato, R.; Hagiwara, Y.; Shiotani, B.; Yamauchi, M.; Nakada, S.; Shibata, A.; Miyagawa, K. Human Rad52 Promotes XPG-Mediated R-loop Processing to Initiate Transcription-Associated Homologous Recombination Repair. Cell 2018, 175, 558-570.e11. [CrossRef]

21. Arauújo, S.J.; Nigg, E.A.; Wood, R.D. Strong Functional Interactions of TFIIH with XPC and XPG in Human DNA Nucleotide Excision Repair, without a Preassembled Repairosome. Mol. Cell. Biol. 2001, 21, 2281-2291. [CrossRef]

22. Thorel, F.; Constantinou, A.; Dunand-Sauthier, I.; Nouspikel, T.; Lalle, P.; Raams, A.; Jaspers, N.G.J.; Vermeulen, W.; Shivji, M.K.K.; Wood, R.D.; et al. Definition of a Short Region of XPG Necessary for TFIIH Interaction and Stable Recruitment to Sites of UV Damage. Mol. Cell. Biol. 2004, 24, 10670-10680. [CrossRef]

23. Mocquet, V.; Lainé, J.P.; Riedl, T.; Yajin, Z.; Lee, M.Y.; Egly, J.M. Sequential recruitment of the repair factors during NER: The role of XPG in initiating the resynthesis step. EMBO J. 2007, 27, 155-167. [CrossRef]

24. Eyboulet, F.; Cibot, C.; Eychenne, T.; Neil, H.; Alibert, O.; Werner, M.; Soutourina, J. Mediator links transcription and DNA repair by facilitating Rad2/XPG recruitment. Genes Dev. 2013, 27, 2549-2562. [CrossRef]

25. Puumalainen, M.-R.; Lessel, D.; Rüthemann, P.; Kaczmarek, N.; Bachmann, K.; Ramadan, K.; Naegeli, H. Chromatin retention of DNA damage sensors DDB2 and XPC through loss of p97 segregase causes genotoxicity. Nat. Commun. 2014, 5, 1-10. [CrossRef]

26. Van Cuijk, L.; Van Belle, G.J.; Turkyilmaz, Y.; Poulsen, S.L.; Janssens, R.C.; Theil, A.F.; Sabatella, M.; Lans, H.; Mailand, N.; Houtsmuller, A.B.; et al. SUMO and ubiquitin-dependent XPC exchange drives nucleotide excision repair. Nat. Commun. 2015, 6, 7499. [CrossRef]

27. Tillhon, M.; Cazzalini, O.; Nardo, T.; Necchi, D.; Sommatis, S.; Stivala, L.A.; Scovassi, A.I.; Prosperi, E. p300/CBP acetyl transferases interact with and acetylate the nucleotide excision repair factor XPG. DNA Repair 2012, 11, 844-852. [CrossRef]

28. Han, C.; Wani, G.; Zhao, R.; Qian, J.; Sharma, N.; He, J.; Zhu, Q.; Wang, Q.-E.; A Wani, A. Cdt2-mediated XPG degradation promotes gap-filling DNA synthesis in nucleotide excision repair. Cell Cycle 2015, 14, 1103-1115. [CrossRef]

29. Bowers, E.M.; Yan, G.; Mukherjee, C.; Orry, A.; Wang, L.; Holbert, M.A.; Crump, N.T.; Hazzalin, C.A.; Liszczak, G.; Yuan, H.; et al. Virtual Ligand Screening of the p300/CBP Histone Acetyltransferase: Identification of a Selective Small Molecule Inhibitor. Chem. Biol. 2010, 17, 471-482. [CrossRef]

30. Scovassi, A.I.; Prosperi, E. Analysis of Proliferating Cell Nuclear Antigen (PCNA) Associated with DNA Excision Repair Sites in Mammalian Cells. In DNA Repair Protocols; Methods in Molecular Biology; Henderson, D.S., Ed.; Humana Press: Totowa, NJ, USA, 2006; Volume 314, pp. 457-475.

31. Cazzalini, O.; Sommatis, S.; Tillhon, M.; Dutto, I.; Bachi, A.; Rapp, A.; Nardo, T.; Scovassi, A.I.; Necchi, D.; Cardoso, M.C.; et al. CBP and p300 acetylate PCNA to link its degradation with nucleotide excision repair synthesis. Nucleic Acids Res. 2014, 42, 8433-8448. [CrossRef]

32. Dutto, I.; Cazzalini, O.; Stivala, L.A.; Prosperi, E. An improved method for the detection of nucleotide excision repair factors at local UV DNA damage sites. DNA Repair 2017, 51, 79-84. [CrossRef]

33. Paunovic, J.; Vucevic, D.; Radosavljevic, T.; Djurdjevic, B.V.; Stankovic, S.; Pantic, I. Effects of Iron Oxide Nanoparticles on Structural Organization of Hepatocyte Chromatin: Gray Level Co-occurrence Matrix Analysis. Microsc. Microanal. $2021,1-8$. [CrossRef]

34. Dancy, B.M.; Cole, P.A. Protein Lysine Acetylation by p300/CBP. Chem. Rev. 2015, 115, 2419-2452. [CrossRef]

35. Shav-Tal, Y.; Blechman, J.; Darzacq, X.; Montagna, C.; Dye, B.T.; Patton, J.G.; Singer, R.H.; Zipori, D. Dynamic Sorting of Nuclear Components into Distinct Nucleolar Caps during Transcriptional Inhibition. Mol. Biol. Cell 2005, 16, 2395-2413. [CrossRef] [PubMed]

36. Mischo, H.E.; Hemmerich, P.; Grosse, F.; Zhang, S. Actinomycin D induces histone gamma-H2AX foci and complex formation of gamma-H2AX with Ku70 and nuclear DNA helicase II. J. Biol. Chem. 2005, 280, 9586-9594. [CrossRef] [PubMed]

37. Furuta, T.; Takemura, H.; Liao, Z.-Y.; Aune, G.; Redon, C.; Sedelnikova, O.A.; Pilch, D.R.; Rogakou, E.P.; Celeste, A.; Chen, H.T.; et al. Phosphorylation of Histone H2AX and Activation of Mre11, Rad50, and Nbs1 in Response to Replication-dependent DNA Double-strand Breaks Induced by Mammalian DNA Topoisomerase I Cleavage Complexes. J. Biol. Chem. 2003, 278 , 20303-20312. [CrossRef]

38. Bensaude, O. Inhibiting eukaryotic transcription. Which compound to choose? How to evaluate its activity? Transcription 2011, 2, 103-108. [CrossRef] 
39. Peltonen, K.; Colis, L.; Liu, H.; Trivedi, R.; Moubarek, M.S.; Moore, H.M.; Bai, B.; Rudek, M.A.; Bieberich, C.; Laiho, M. A Targeting Modality for Destruction of RNA Polymerase I that Possesses Anticancer Activity. Cancer Cell 2014, 25, 77-90. [CrossRef]

40. Wienholz, F.; Vermeulen, W.; Marteijn, J.A. Amplification of unscheduled DNA synthesis signal enables fluorescence-based single cell quantification of transcription-coupled nucleotide excision repair. Nucleic Acids Res. 2017, 45, e68. [CrossRef] [PubMed] 